\title{
Prevalence of Mupirocin Resistance in Methicillin Resistant Staphylococcus aureus Strains isolated from a Tertiary Care Hospital
}

\author{
B. Madhumati*, Vijaya Rajendran and K. Ashwin \\ Department of Microbiology, East point college of Medical Sciences and Research Centre, \\ Avalahalli, Bangalore 49, Karnataka, India \\ *Corresponding author
}

\begin{tabular}{l} 
K e y w o r d s \\
$\begin{array}{l}\text { Methicillin resistant } \\
\text { Staphylococcus aureus } \\
\text { (MRSA), Mupirocin, }\end{array}$ \\
$\begin{array}{l}\text { Clinical and Laboratory } \\
\text { Standards Institute } \\
\text { (CLSI). The European }\end{array}$ \\
$\begin{array}{l}\text { Committee on } \\
\text { Antimicrobial } \\
\text { Susceptibility Testing } \\
\text { (EUCAST), Macrolide- } \\
\text { lincosamide } \\
\text { streptogramin B (MLSB) } \\
\text { phenotype }\end{array}$ \\
\hline $\begin{array}{l}\text { Article Info } \\
\text { Accepted: } \\
\text { 04 April } 2018 \\
\text { Available Online: } \\
10 \text { May } 2018\end{array}$ \\
\hline
\end{tabular}

A B S T R A C T

Mupirocin is a topical antibiotic that has been used extensively for treating methicillin resistant Staphylococcus aureus (MRSA) associated infections. However, the prevalence of resistance to mupirocin is being increasingly found due to its irrational and widespread use. Retapamulin: a pleuromutilin antibacterial agent is an effective topical antibiotic against these resistant strains. This study was carried out to determine the rates of resistance to mupirocin in MRSA isolates in our patient population, and to estimate the association of such resistance and resistance to other classes of antimicrobial agents in order to consider the effect of the use of mupirocin on the selection of antimicrobial resistant strains. This prospective study was conducted in the Department of Microbiology at VIMS \& RC, Bengaluru. 200 Staphylococcus aureus isolates were recovered from various clinical specimens from out patients and in patients admitted into various wards and intensive care units. Isolation and identification of isolates $S$. aureus isolated from clinical specimens were identified according to the standard laboratory operating procedures. Antimicrobial sensitivity was determined by the Vitek $2 \mathrm{~K}$ automated system. All tests and quality assurance procedures were performed and interpreted according to the standards set by the Clinical and Laboratory Standards Institute (CLSI). For fusidic acid and retapamulin The European Committee on Antimicrobial Susceptibility Testing (EUCAST) criteria was used. For the macrolide-lincosamide streptogramin B (MLSB) phenotype (inducible clindamycin resistance encoded by the plasmid-borne gene, erm) was determined by the disc approximation test, clindamycin was reported to be resistant. Mupirocin resistance testing was done by disc diffusion with disc concentration of $5 \mu \mathrm{g}$ and $200 \mu \mathrm{g}$. Among the 200 Staphylococcus aureus isolates 108 (54\%) were MRSA, highest percentage of MRSA strains were from blood isolates (62\%), followed by aspirated fluids and pus (59\% and $56 \%$ respectively). Of the 108 MRSA strains, 26 (24\%) were mupirocin resistant Staphylococcus aureus (MupRSA). Mupirocin resistance was not detected in methicillin sensitive Staphylococcus aureus (MSSA) isolates. High-level mupirocin resistance was observed in $11 \%$ and low-level resistance in $13 \%$ of the 108 isolates. Higher prevalence of high level mupirocin resistance was from blood isolates followed by pus ( $45 \%$ and $36.3 \%$ respectively), low level mupirocin resistance was maximum seen in the isolates from respiratory secretions $(46 \%)$. MupRSA was more frequently isolated from ICUs and surgical wards. The mupirocin resistant MRSA strains exhibited resistance to other class of antibiotics also: ampicillin (90\%), ciprofloxacin (88\%), erythromycin (86\%), co trimoxazole (73\%). However fusidic acid, vancomycin and linezolid showed sensitivity of $99 \%, 96 \%$ and $96 \%$ respectively. All the strains were sensitive to retapamulin. Increase in mupirocin resistance among MRSA isolates is a matter of concern. Antimicrobial stewardship programmes are important to address excessive or inappropriate antimicrobial usage. Alternative agents to mupirocin should be considered to counteract the clinical failure of decolonization regimens and to prevent the selection of multiple resistant strains. 


\section{Introduction}

Staphylococcus aureus is a major pathogen responsible for various community-acquired and nosocomial infections, including bacteraemia, pneumonia, skin and soft tissue infections, and osteomyelitis. Methicillinresistant S. aureus (MRSA) are implicated in serious infections and nosocomial outbreaks. These strains show resistance to a wide range of antibiotics, thus limiting the treatment options to very few agents such as glycopeptides and linezolid (Xavier Malaviolle et al., 2008).

Carriage of MRSA in nose, axilla, perineum and hands of patients and health care personnel is an important risk factor for MRSA acquisition and spread. Decolonization from the site of carriage is one of the modalities for prevention of MRSA infections in healthcare settings. Mupirocin (pseudomonic acid A) derived from Pseudomonas fluorescens is an important topical antibiotic ointment for the nasal decolonization of MRSA in carriers. It acts by binding specifically to the bacterial isoleucyltRNA synthetase (IRS) enzyme and inhibits its protein synthesis. Along with its use as a decolonising agent in health care personnel and patients, it has also been used for treatment of staphylococcal skin and soft tissue infections (Singh Amit et al., 2013; Summiya Nizamuddin et al., Hetem and Bonten, 2013).

Resistance to mupirocin is being increasingly found due to its irrational use, which leads to improper decolonization of MRSA from the site of carriage and spread of infection.

The mechanisms of mupirocin resistance have been elucidated, low-level resistance can be caused by an alteration in the isoleucyl t RNA sythetase gene, ileS. This mutation is stable and non-transferrable. High-level resistance is mostly associated with the presence of the тирA gene, which encodes an alternate isoleucyl-tRNA synthetase. High-level resistance in the absence of this gene has been encountered, suggesting resistance by other mechanisms furthermore, the тирA gene is associated with mobile genetic elements and is mostly plasmid borne, which may facilitate the spread of this resistance mechanism. These plasmids also carry resistance genes to other antimicrobial agents, such as the macrolides, gentamicin, tetracycline and trimethoprim. Although sensitivity to mupirocin is not affected by the same genetic elements as resistance to beta-lactam agents, such as cloxacillin, an association between MRSA and mupirocin resistance has been noted in the literature (Ravisekhar Gadepalli et al., 2007; Wasserman et al., 2014).

Mupirocin-resistant strains have been grouped into two distinct phenotypes: low-level resistance (MuL) with MICs of 8-256 $\mu \mathrm{g} / \mathrm{ml}$, and high-level resistance (MuH) with MICs $\geq 512 \mu \mathrm{g} / \mathrm{ml}$. An isolate with MIC $\leq 4 \mu \mathrm{g} / \mathrm{ml}$ is considered as mupirocin-sensitive. With the previously used $5 \mu \mathrm{g}$ mupirocin disk, MuL and $\mathrm{MuH}$ strains cannot be differentiated. However it can be performed by concomitant use of $5 \mu \mathrm{g}$ and $200 \mu \mathrm{g}$ mupirocin disks. $\mathrm{MuH}$ strains have been found to be associated with failure of mupirocin as a decolonising agent as well as for treatment of skin and soft tissue infections. Plasmid-mediated mирA encoding a novel isoleucyl RNA synthetase is a major genetic mechanism seen in high-level mupirocin resistance isolates. Whereas base pair changes in native isoleucyl RNA synthetase gene is seen in low-level mupirocin resistance isolates (Singh Amit et al., 2013).

The presence of the mирA gene can be confirmed by polymerase chain reaction (PCR), but this may be prohibitively expensive when large numbers of isolates are to be screened, and the other mechanisms of 
resistance also cannot be excluded (Wasserman et al., 2014).

Retapamulin: a pleuromutilin antibacterial agent is an effective topical antibiotic against these resistant strains. Currently it is used against a variety of Gram positive pathogens associated with secondarily-infected traumatic lesions and secondarily-infected dermatoses. The pleuromutilins are potent inhibitors of protein synthesis in bacteria through the interference of peptide bond formation by binding to the peptidyl transferase center of the 50S ribosomal subunit1 Due to the unique pleuromutilin mode of action, retapamulin shows no target specific cross-resistance to other classes of antibacterials (Dhingra et al., 2013; Ronald N. Jones et al., 2006).

Though mupirocin resistance is often associated with methicillin resistance, however the true extent of mupirocin resistance in our area is unknown.

Thus, this study was carried out to determine the rates of high-level and low level resistance to mupirocin in MRSA isolates in our patient population, and to estimate the association of such resistance and resistance to other classes of antimicrobial agents in order to consider the effect of the use of mupirocin on the selection of antimicrobial resistant strains.

\section{Materials and Methods}

This prospective study was conducted in the Department of Microbiology at VIMS \& RC.

Staphylococcus aureus isolates were recovered from clinical specimens comprising of pus, respiratory secretions, catheter tip, aspirated fluids and blood from out patients and in patients admitted into various wards and intensive care units.

Isolates from urine were not included.
Isolation and identification of isolates $S$. aureus isolated from clinical specimens was identified according to the standard laboratory operating procedures. Antimicrobial sensitivity was determined by the Vitek $2 \mathrm{~K}$ automated system. All tests and quality assurance procedures were performed and interpreted according to the standards set by the Clinical and Laboratory Standards Institute (CLSI). For fusidic acid and retapamulin The European Committee on Antimicrobial Susceptibility Testing (EUCAST) criteria was used (EUCAST and CLSI potency NEOSENSITABS). For the macrolide-lincosamide streptogramin B (MLSB) phenotype (inducible clindamycin resistance encoded by the plasmid-borne gene, erm) was determined by the disc approximation test, clindamycin was reported to be resistant. Moxifloxacin was used as a marker for fluoroquinolone resistance. Mupirocin resistance testing was done by disc diffusion with disc concentration of $5 \mu \mathrm{g}$ and $200 \mu \mathrm{g}$. Zone diameter of $>14 \mathrm{~mm}$ for both disks was taken as susceptible for mupirocin. Whereas, isolates that showed zone diameters $<14 \mathrm{~mm}$ in the $5 \mu \mathrm{g}$ disk but $>$ to $14 \mathrm{~mm}$ in the $200 \mu \mathrm{g}$ disk were considered to be low-level mupirocin resistant strains. All isolates with zone diameters $<14 \mathrm{~mm}$ for both $5 \mu \mathrm{g}$ and $200 \mu \mathrm{g}$ disks were considered to be high-level mupirocin resistant strains.

\section{Results and Discussion}

In this study 200 Staphylococcus aureus isolates were obtained from various clinical specimens such as pus $(\mathrm{n}=88)$, respiratory secretions $(n=51)$, aspirated fluids $(n=27)$, blood $(n=21)$ and others $(n=13)$ (Table 1).

Among the 200 Staphylococcus aureus isolates 108 (54\%) were MRSA, highest percentage of MRSA strains were from blood isolates (62\%), followed by aspirated fluids and pus (59\% and $56 \%$ respectively) (Table $1)$. 
Table.1 Isolation of Staphylococcus aureus and MRSA from various clinical samples

\begin{tabular}{|c|c|c|c|c|}
\hline SI. no & Clinical samples & $\begin{array}{l}\text { Staphylococcus aureus } \\
\text { isolates }\end{array}$ & MRSA strains & $\%$ \\
\hline 1 & Pus & 88 & 49 & 56 \\
\hline 2 & Respiratory secretions & 51 & 27 & 53 \\
\hline 3 & Aspirated fluids & 27 & 16 & 59 \\
\hline 4 & Blood & 21 & 13 & 62 \\
\hline 5 & Others & 13 & 3 & 23 \\
\hline & Total & 200 & 108 & 54 \\
\hline
\end{tabular}

Table.2 Prevalence of mupirocin resistance in MRSA strains

MRSA strains 108
Mupirocin sensitive n (\%)

$82(76)$
Mupirocin resistance n (\%) $26(24)$

Table.3 Isolation of MuH and MuL in MRSA strains from various clinical samples

\begin{tabular}{|c|l|c|c|c|}
\hline Sl.no & \multicolumn{1}{|c|}{ Clinical samples } & MRSA strains & $\begin{array}{c}\text { MuH } \\
\text { n (\%) }\end{array}$ & $\begin{array}{c}\text { MuL } \\
\text { n }(\%)\end{array}$ \\
\hline 1 & Pus & 49 & $4(33.3)$ & $3(21.4)$ \\
\hline 2 & Respiratory secretions & 27 & $2(17)$ & $6(43)$ \\
\hline 3 & Aspirated fluids & 16 & $1(8.3)$ & $3(21.4)$ \\
\hline 4 & Blood & 13 & $5(42)$ & $1(7)$ \\
\hline 5 & Others & 3 & 0 & $1(7)$ \\
\hline & Total & $\mathbf{1 0 8}$ & $\mathbf{1 2 ( 1 1 )}$ & $\mathbf{1 4}(\mathbf{1 3})$ \\
\hline
\end{tabular}

Table.4 Antimicrobial resistance pattern of MRSA and MupRSA against various antibiotics tested

\begin{tabular}{|c|l|c|c|}
\hline Sl.no & \multicolumn{1}{|c|}{ Antibiotic } & MIRA (\%) & MuRSA (\%) \\
\hline $\mathbf{1}$ & Erythromycin & 80 & 86 \\
\hline $\mathbf{2}$ & Clindamycin & 51 & 65 \\
\hline $\mathbf{3}$ & Co-trimoxazole & 65 & 73 \\
\hline $\mathbf{4}$ & Linezolid & 2 & 4 \\
\hline $\mathbf{5}$ & Tetracycline & 60 & 82 \\
\hline $\mathbf{6}$ & Vancomycin & 1 & 4 \\
\hline $\mathbf{7}$ & Rifampicin & 10 & 24 \\
\hline $\mathbf{8}$ & Chloramphenicol & 21 & 36 \\
\hline $\mathbf{9}$ & Ciprofloxacin & 53 & 88 \\
\hline $\mathbf{1 0}$ & Gentamycin & 63 & 76 \\
\hline $\mathbf{1 1}$ & Ampicillin & 86 & 90 \\
\hline $\mathbf{1 2}$ & Fusidic acid & 0 & 1 \\
\hline $\mathbf{1 3}$ & Retapamulin & 0 & 0 \\
\hline
\end{tabular}


Of the 108 MRSA strains, 26 (24\%) were mupirocin resistant Staphylococcus aureus (MupRSA) (Table 2). Mupirocin resistance was not detected in methicillin sensitive Staphylococcus aureus (MSSA) isolates. High-level mupirocin resistance was observed in $11 \%$ and low-level resistance in $13 \%$ of the 108 isolates.

Higher prevalence of high level mupirocin resistance was from blood isolates followed by pus ( $45 \%$ and $36.3 \%$ respectively), low level mupirocin resistance was maximum seen in the isolates from respiratory secretions (46\%) (Table 3).

MupRSA was more frequently isolated from ICUs and surgical wards.

The mupirocin resistant MRSA strains exhibited resistance to other class of antibiotics also: ampicillin (90\%), ciprofloxacin $(88 \%)$, erythromycin $(86 \%)$, co trimoxazole (73\%). However fusidic acid, vancomycin and linezolid showed sensitivity of $99 \%, 96 \%$ and $96 \%$ respectively (Table 4).

All the strains were sensitive to retapamulin.

200 Staphylococcus aureus isolates were obtained from various clinical specimens, among them 108 (54\%) were MRSA, highest percentage of MRSA strains were from blood isolates (62\%), followed by aspirated fluids and pus (59\% and $56 \%$ respectively).

Of the 108 MRSA strains 26 (24\%) were mupirocin resistant and $82(76 \%)$ were mupirocin sensitive

Higher prevalence of high level mupirocin resistance $(\mathrm{MuH})$ was from blood isolates followed by pus $(42 \%$ and $33.3 \%$ respectively), low level mupirocin resistance (MuL) was maximum seen in the isolates from respiratory secretions (43\%).
The mupirocin resistant MRSA strains exhibited resistance to various classes of antibiotics: ampicillin (90\%), ciprofloxacin (88\%), erythromycin $86 \%$ and co trimoxazole $73 \%$. However fusidic acid, vancomycin and linezolid showed sensitivity of $99 \%, 96 \%$ and $96 \%$ respectively.

All the strains were sensitive to retapamulin.

Mupirocin (pseudomonic acid A) derived from Pseudomonas fluorescens is widely used topical antibiotic for the treatment of MRSAassociated infections. In healthcare institute it is used for nasal decolonization of health care personnel to prevent the spread of MRSA among co-workers and the patients. Emergence of resistance to mupirocin is likely to worsen the problem. Studies suggest mupA gene which encodes mupirocin resistance is transferred from commensal flora of skin to MRSA during mupirocin therapy. This could be a threat to irrational use of mupirocin as it may lead to the development and spread of mupirocin resistance (Singh Amit et al., 2013; Charan Kaur Dardi, 2014). The prevalence of high-level mupirocinresistant MRSA in the present study was $11 \%$ and low-level resistance was $13 \%$.

This is comparable to reports in the literature of $1-13 \%$ for low-level and $2.4-14 \%$ for highlevel resistance by various authors (Vasquez et al., 2000; Banerjee John et al., 2013; Banerjee John et al., 2013). None of the MSSA were mupirocin resistant.

Various studies suggest that during mupirocin prophylaxis, transfer of тирA gene from normal commensal flora of skin as in Staphylococcus epidermidis to MRSA is responsible for the emergence of mupirocin resistance (Nonika Rajkumari et al., 2014; Hurdle et al., 2005). Therefore the sensitivity to mupirocin should be confirmed before it can be used as a decontaminating agent and 
should be factored into local infection control policies.

The majority of the resistant isolates were from the surgical disciplines, ICUs and in patients as they deal with the majority of complicated soft tissue and skeletal infections from which $S$. aureus is the most frequently isolated pathogen.

The link between antimicrobial consumption and antimicrobial resistance profiles is well established. Therefore, collections of isolates representing invasive infection (blood culture, pus isolates) and nosocomial infection have a denser antimicrobial history, resulting in the resistance to antimicrobial agents.

Mupirocin-resistant MRSA isolates showed higher antibiotic resistance to several classes of antimicrobial agents, i.e. MLSB phenotypes, quinolones, co-trimoxazole, and rifampicin, however they showed sensitivity to ritapamulin, vancomycin, linezolid and fusidic acid, which is in comparison with the various authors around the world (Banerjee John et al., 2013; Banerjee John et al., 2013).

This is biologically plausible as genetic material encoding for resistance to different classes of antimicrobial agents may be carried on the same mobile genetic elements. It is a cause for concern as it implies that mupirocin use will lead to environmental pressure for the selection of resistance to other classes of antimicrobial agents, and vice versa (Wasserman et al., 2014).

For systemic treatment of MRSA, fusidic acid has proven useful when combined with agents such as vancomycin, but not as monotherapy (Malaviolle et al., 2008). Hydrogen peroxide cream also has been recommended as a topical alternative to mupirocin or perhaps the newer ritapamulin has also seen in this study (Christensen and Anehus, 1994).
Genotypic methods such as PCR can be used as the final confirmatory test for detection of mupirocin-resistant MRSA isolates. The lack of a confirmatory test is a limitation of the present study.

Increase in mupirocin resistance among MRSA isolates is a matter of concern. The better understanding of the mechanisms, clinical significance and epidemiology of mupirocin resistance is important for predicting how changes in mupirocin use may affect the emergence of mupirocin resistance. Hence it is recommended that routine testing of MRSA for mupirocin resistance be conducted which facilitates the early detection of resistance and assists in the control and spread of mupirocin-resistant MRSA.

Antimicrobial stewardship programmes are important to address excessive or inappropriate antimicrobial usage. It is important to look beyond the usage of systemic antimicrobial agents and to adopt a more comprehensive approach to decolonization and environmental stewardship.

Alternative agents to mupirocin should be considered to counteract the clinical failure of decolonization regimens and to prevent the selection of multiple resistant strains.

\section{Approved by ethical committee}

\section{Conflict of interest}

The authors declare that there are no conflicts of interest

\section{References}

Banerjee John JW, Routray A, Madhavan R. Mupirocin Resistance in Methicillin Resistant Staphylococcus aureus. Int J Pharm Biol Sci. 2013; 4: 858-61. 
Banerjee John, J.W., Abhisek Routray and Radha Madhavan. Mupirocin resistance in methicillin resistant Staphylococcus aureus. Int J Pharm Bio Sci. 2013 July; 4(3): (B) $858-861$.

Charan Kaur Dardi. Mupirocin resistance in clinical isolates of methicillin resistant Staphylococcus aureus from a tertiary care rural hospital. International Journal of Advanced Medical and Health Research. Volume 1; Issue 2: Jul-Dec 2014.

Christensen OB, and Anehus S. Hydrogen peroxide cream: An alternative to topical antibiotics in the treatment of impetigo contagiosa. Acta Derm Venereol. 1994; 74: 460-2.

Clinical and Laboratory Standards Institute (CLSI), Performance Standards for Antimicrobial Susceptibility Testing. Approved Standards M100-S.

Dhingra, D., A. Parakh, S. Ramachandran. Retapamulin: A newer topical antibiotic. JPGM. 2013; Vol 5: 127-130.

EUCAST - and CLSI potency NEOSENSITABS. Document: 1.4.0, page 1/1. ROSCO diagnostic.

Hetem, D.J., and M.J.M. Bonten. Clinical relevance of mupirocin resistance in Staphylococcus aureus. Journal of Hospital Infection. 2013; 85: 249-256.

Hurdle JG, O'Neill AJ, Mody L, Chopra I, Bradley SF. In vivo transfer of highlevel mupirocin resistance from Staphylococcus epidermidis to methicillin-resistant Staphylococcus aureus associated with failure of mupirocin prophylaxis. J Antimicrob Chemother. 2005; 56:1166-8.

Malaviolle X, Nonhoff C, Denis O, Rottiers $\mathrm{S}$, Struelens MJ. Evaluation of disc diffusion methods and Vitek 2 automated system for testing susceptibility to mupirocin in Staphylococcus aureus. J Antimicrob Chemother. 2008; 62: 1018-23.
Nonika Rajkumari, Purva Mathur, Nidhi Bhardwaj, Gunjan Gupta, Rajrani Dahiya, Bijayini Behera1, Mahesh Chandra Misra. Resistance pattern of mupirocin in methicillin - resistant Staphylococcus aureus in trauma patients and comparison between disc diffusion and $\mathrm{E}^{-}$test for better detection of resistance in low resource countries. Journal of Laboratory Physicians. Jul-Dec 2014; Vol-6: Issue2.

Ravisekhar Gadepalli, Benu Dhawan, Srujana Mohanty, Arti Kapil. Mupirocin resistance in Staphylococcus aureus in an Indian hospital. Diagnostic Microbiology and Infectious Disease. 2007; 58: 125-127.

Ronald N. Jones, Thomas R. Fritsche, Helio S. Sader, and James E. Ross. Activity of Retapamulin (SB-275833), a Novel Pleuromutilin, against Selected Resistant Gram-Positive Cocci. Antimicrobial agents and chemotherapy. July 2006; p. 25832586.

Singh Amit K, Venkatesh Vimala, Singh Mastan. Mupirocin resistance in clinical isolates of Staphylococcus aureus in a tertiary care hospital set up in north. Int J Med Res Health Sci. 2013; 2(4): 840847.

Summiya Nizamuddin, Seema Irfan, Afia Zafar. Evaluation of prevalence of low and high level Mupirocin resistance in Methicillin Resistant Staphylococcus aureus isolates at a tertiary care hospital. J Pak Med Assoc. 61(6); 51921.

Vasquez JE, Walker ES, Franzus BW, Overbay BK, Reagan DR, Sarubbi FA. The epidemiology of mupirocin resistance among methicillin-resistant Staphylococcus aureus at a veteran's affairs hospital. Infect Control Hosp Epidemiol. 2000; 21:459-64. 
Wasserman, E., H Orth, M Senekal, K Harvey. High prevalence of mupirocin resistance associated with resistance to other antimicrobial agents in Staphylococcus aureus isolated from patients in private health care, Western Cape. South Afr J Infect Dis. 2014; 29 (4):126-132.
Xavier Malaviolle, Claire Nonhoff, Olivie Denis, Sylvianne Rottiers and Marc J. Struelens. Evaluation of disc diffusion methods and Vitek 2 automated system for testing susceptibility to mupirocin in Staphylococcus aureus. Journal of Antimicrobial Chemotherapy. 2008; 62: 1018-1023.

\section{How to cite this article:}

Madhumati, B., Vijaya Rajendran and Ashwin, K. 2018. Prevalence of Mupirocin Resistance in Methicillin Resistant Staphylococcus aureus Strains isolated from a Tertiary Care Hospital. Int.J.Curr.Microbiol.App.Sci. 7(05): 329-336. doi: https://doi.org/10.20546/ijcmas.2018.705.042 